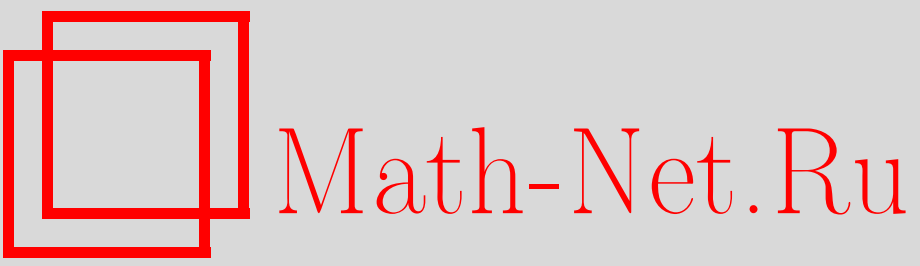

Ю. П. Вирченко, А. В. Субботин, Описание класса эволюционных уравнений ферродинамики, Итоги науки и техн. Сер. Соврем. мат. и ее прил. Темат. обз., 2019, том 170, 15-30

DOI: https://doi.org/10.36535/0233-6723-2019-170-15-30

Использование Общероссийского математического портала Math-Net.Ru подразумевает, что вы прочитали и согласны с пользовательским соглашением

http: //www. mathnet.ru/rus/agreement

Параметры загрузки:

IP: 54.197 .217 .227

26 апреля 2023 г., $17: 36: 27$ 


\title{
ОПИСАНИЕ КЛАССА ЭВОЛЮЦИОННЫХ УРАВНЕНИЙ ФЕРРОДИНАМИКИ
}

\author{
(c) 2019 г. Ю. П. ВИРЧЕНКО, А. В. СУББОТИН \\ Посвящается академику НАНУ С. В. Пелетминскому
}

\begin{abstract}
АннотАция. Формулируется постановка задачи, которая возникает в связи проблемой конструирования эволюционных уравнений для описания динамики конденсированных сред, обладающих внутренней структурой. В рамках этой постановки дается описание класса всех эволюционных уравнений для векторного и псевдовекторного полей на $\mathbb{R}^{3}$ с инфинитизимальным сдвигом, определяемым дифференциальным оператором второго порядка дивергентного типа, инвариантным относительно трансляций $\mathbb{R}^{3}$, трансляций времени и преобразующихся ковариантным образом при вращениях $\mathbb{R}^{3}$. Отдельно исследован случай уравнений этого класса, сохраняющих соленоидальность и унимодальность поля. Установлена общая формула для всех операторов, соответствующих этим уравнениям.
\end{abstract}

Ключевые слова: дивергентный дифференциальный оператор, псевдовекторное поле, плотность потока поля, унимодальность, соленоидальность, уравнение ферродинамики.

\section{DESCRIPTION OF A CLASS OF EVOLUTIONARY EQUATIONS IN FERRODYNAMICS}

\author{
(c) 2019 YU. P. VIRCHENKO, A. V. SUBBOTIN
}

\begin{abstract}
In this paper, we state the problem of constructing evolution equations describing the dynamics of condensed matter with an internal structure. Within the framework of this statement, we describe the class of evolution equations for vector and pseudovector fields on $\mathbb{R}^{3}$ with an infinitesimal shift defined by a second-order, divergent-type differential operator, which is invariant under translations of $\mathbb{R}^{3}$ and time translations and is transformed covariantly under rotations of $\mathbb{R}^{3}$. The case of equations of this class with preserved solenoidality and unimodality of the field is studied separately. A general formula for all operators corresponding to these equations is established.
\end{abstract}

Keywords and phrases: divergent differential operator, pseudovector field, flux density, unimodality, solenoidality, ferrodynamic equation.

AMS Subject Classification: 35Q60, 35K10

1. Введение. При теоретических исследованиях физических задач, для решения которых привлекаются дифференциальные уравнения в частных производных, ключевым вопросом является следующий: на основе каких физически оправданных положений могут быть сконструированы используемые уравнения, подходящие для достижения целей исследования? Обычно рассуждения, применяемые при построении дифференциальных уравнений, адекватных описываемой физической реальности, не являются объектом исследования собственно теории дифференциальных уравнений; такая ситуация сложилась исторически и связана с тем, что наиболее известные и используемые уравнения математической физики - такие как, например, уравнение теплопроводности, система уравнений гидродинамики ньютоновских жидкостей, система уравнений Максвелла 
и т. д. - являются в настоящее время в достаточной степени обоснованными с физической точки зрения, и те математические проблемы, которые приходилось решать их первооткрывателям, не очень важны с математической точки зрения.

Положение совершенно меняется, когда физикам-теоретикам приходится решать задачи о «выводе» адекватных дифференциальных уравнений при исследовании физических ситуаций, принципиально отличных от уже хорошо изученных. При этом часто хорошо отработанные стереотипы рассуждений, такие, как, например, лагранжев (гамильтонов) формализм (см., например, $[1,5,7,8])$, не приводят к однозначному результату. Эта ситуация возникает, например, при описании динамики конденсированных сред, обладающих сложной внутренней структурой, мгновенное физическое состояние которых в каждой пространственной точке с радиус-вектором $\boldsymbol{x} \in \mathbb{R}^{3}$ характеризуется набором значений термодинамических параметров $X_{a}, a=1, \ldots, N$.

Пусть возможные значения параметров $X_{a}, a=1, \ldots, N$, принадлежат некоторой области линейного пространства $\mathcal{L}$ размерности $N$. Тогда состояние среды в каждый момент времени описывается набором зависящих от времени $t \in \mathbb{R}_{+}$полей $\boldsymbol{X}(\boldsymbol{x}, t)=\left\langle X_{a}(\boldsymbol{x}, t), a=1, \ldots, N\right\rangle$, на $\mathbb{R}^{3}$. Изменение же состояния среды с течением времени описывается системой эволюционных уравнений

$$
\dot{X}_{a}(\boldsymbol{x}, t)=\left(\mathrm{L}_{a}[\boldsymbol{X}]\right)(\boldsymbol{x}, t), \quad a=1, \ldots, N,
$$

которому этот набор полей подчинен. Здесь точка обозначает дифференцирование по $t$ и $\mathrm{L}_{a}-$ дифференциальный оператор порядка $s$, в общем случае нелинейный. Он осуществляет отображение $\mathrm{L}_{a}:\left[C_{s, \text { loc }}\left(\mathbb{R}^{3}\right)\right]^{N} \rightarrow\left[C_{s, \text { loc }}\left(\mathbb{R}^{3}\right)\right]^{N}$ и представляется набором вектор-функций

$$
\left(\mathrm{L}_{a}[\boldsymbol{X}]\right)(\boldsymbol{x}, t)=L_{a}(\boldsymbol{X}, \nabla \otimes \boldsymbol{X}, \ldots, \underbrace{\nabla \otimes \cdots \otimes \nabla}_{s} \otimes \boldsymbol{X} ; \boldsymbol{x}, t), \quad a=1, \ldots, N,
$$

принадлежащих $\left[C_{s, \text { loc }}\left(\mathcal{L} \times\left(\mathbb{R}^{3} \times \mathcal{L}\right) \times \cdots \times\left(\mathbb{R}^{3^{s}} \times \mathcal{L}\right) \times \mathbb{R}^{3}\right)\right]^{N}$, от компонент набора $\boldsymbol{X}(\boldsymbol{x}, t)$ полей и их векторных производных $\left(\boldsymbol{\otimes}^{n} \nabla\right) \otimes \boldsymbol{X}(\boldsymbol{x}, t), n=1, \ldots, s$. Функции $X_{a}(\boldsymbol{x}, t), a=1, \ldots, N$, подразумеваются $s$ раз непрерывно дифференцируемыми по компонентам вектора $\boldsymbol{x}$, чтобы применение оператора $L_{a}$ имело смысл. Здесь и далее $\nabla$-операция градиента в $\mathbb{R}^{3}$. Обозначим линейное многообразие всех таких операторов посредством $\mathcal{K}_{s}(\mathcal{L})$.

Проблема, которая должна быть решена физиком, состоит в адекватном конструировании оператора $\mathrm{L}_{a}$. При ее решении допустимо руководствоваться только наиболее общими физическими принципами. Кроме того, возникает необходимость построить дифференциальный оператор $\mathrm{L}_{a}$ так, чтобы решения системы (1.1) наверняка подчинялись определенным условиям, которые выделяют в пространстве $\left[C_{s, l o c}\left(\mathbb{R}^{3}\right)\right]^{N}$ некоторое дифференцируемое многообразие $\mathcal{M} \subset\left[C_{s, \text { loc }}\left(\mathbb{R}^{3}\right)\right]^{N}$. В таком случае возникает достаточно содержательная математическая задача. Она состоит в описании всего класса дифференциальных операторов, удовлетворяющих указанным общим принципам и дополнительным ограничениям. Решение этой задачи дает возможность выбора на практике подходящего дифференциального оператора из указанного класса.

В настоящей работе мы не будем интересоваться корректностью постановки каких-либо начально-краевых задач для уравнений (1.1), определяемых операторами класса $\mathcal{K}_{s}(\mathcal{L})$. Нас будет интересовать только лишь вопрос, удовлетворяют ли дифференциальные операторы $\mathrm{L}_{a}$ этого класса определенным требованиям общего характера, которые диктуются физической природой задачи.

По самому смыслу физической ситуации, для описания которой предназначаются уравнения (1.1), потребуем, чтобы оператор $L_{a}$ был инвариантен по отношению группы преобразований $\mathbf{T} \otimes \mathbf{R}^{3}$, где $\mathbf{T}$ - группа трансляций по переменной $t, \mathbf{R}^{3}$ - группа трансляций пространства $\mathbb{R}^{3}$, а также требованию ковариантности по отношению к группе $\mathbf{O}_{3}$ вращений пространства $\mathbb{R}^{3}$. Естественно, при этом предполагается, что линейное пространство $\mathcal{L}$ преобразуется по представлению группы $\mathbf{O}_{3}$.

Так как в дальнейшем нас будут интересовать не сами представления группы $\mathbf{O}_{3}$, а пространства, где они действуют, то мы будем называть пространство $\mathcal{L}$ линейным представлением, что 
не будет вызывать недоразумений. В общем случае, важном с точки зрения физических приложений, это представление разлагается на произведение неприводимых спин-тензорных представлений (см. [11]) и набор скаляров (псевдоскаляров), неизменных при вращениях $\mathbb{R}^{3}$.

Что касается условия инвариантности относительно групп $\mathbf{T}$ и $\mathbf{R}^{3}$, то оно удовлетворяется в том и только в том случае, когда вектор-функции $L_{a}, a=1, \ldots, N$, не зависят явно ни от $t$, ни от $\boldsymbol{x}$. Требование же ковариантности накладывает уже довольно значительные ограничения на общий вид этих вектор-функций. Его описание приводит к довольно обширному линейному многообразию $\mathcal{K}_{s}(\mathcal{L})$. Существенное сужение этого многообразия происходит при введении упомянутых выше дополнительных ограничений, т.е. при сужении действия оператора на подходящее многообразие $\mathcal{M}$. Это оказывается важным с точки зрения востребованности решения сформулированной задачи в физических приложениях. Дополнительно, существенное упрощение происходит в том случае, если физическая среда не является анизотропной. Тогда представление $\boldsymbol{X}$ не содержит постоянного тензора второго ранга, описывающего анизотропию. В таком случае мы будем называть ее сферически симметричной.

$\mathrm{B}$ настоящей работе мы ограничимся решением задачи об описании многообразия $\mathcal{K}_{2}\left(\mathbb{R}^{3}\right)$, $N=3$, в том случае, когда $\mathrm{L}_{a}$ является дифференциальным оператором второго порядка дивергентного типа. При этом мы рассмотрим случаи, когда поле $\boldsymbol{X}$ является как векторным, так и псевдовекторным (см., например, [13]). Более того, исследуем случай, когда поле $\boldsymbol{X}$ подчинено дополнительным ограничениям: условиям унимодальности $\boldsymbol{X}^{2}(\boldsymbol{x}, t)=$ const и соленоидальности $(\nabla, \boldsymbol{X})=0$. Эти условия выделяют многообразие $\mathcal{M}_{0}$, на котором эволюционное уравнение (1.1) заведомо обладает такими инвариантами движения. Решение этой задачи является востребованным с точки зрения приложений к описанию динамики твердотельной среды с собственным электрическим моментом (векторный случай) и среды, упорядоченной ферромагнитным образом (псевдовекторный случай).

2. Задача описания многообразия операторов $L_{a}$ дивергентного типа. Согласно общему определению дивергентных дифференциальных операторов (см., например, [6]), в рассматриваемом случае, когда дифференциальный оператор $\mathrm{L}_{a}$ определяется вектор-функцией $L_{a}$, $a=1, \ldots, N$, инвариантной относительно преобразований из $\mathbf{T} \otimes \mathbf{R}^{3}$ и ковариантной относительно преобразований группы $\mathbf{O}_{3}$, будем называть уравнение (1.1) уравнением дивергентного типа, если вектор-функция $L_{a}$ имеет вид

$$
L_{a}(\boldsymbol{X}, \nabla \otimes \boldsymbol{X}, \ldots, \underbrace{\nabla \otimes \cdots \otimes \nabla}_{s} \otimes \boldsymbol{X})=\nabla_{k} S_{a ; k}, \quad a=1, \ldots, N
$$

где

$$
S_{a ; k} \equiv S_{a ; k}(\boldsymbol{X}, \nabla \otimes \boldsymbol{X}, \ldots, \underbrace{\nabla \otimes \cdots \otimes \nabla}_{s} \otimes \boldsymbol{X}) \in\left[C_{1, \operatorname{loc}}\left(\mathcal{L} \times \cdots \times\left(\mathbb{R}^{3 s} \times \mathcal{L}\right)\right)\right]^{3 N}
$$

- вектор-функция со значениями в $\mathcal{L} \times \mathbb{R}^{3}$, компоненты которой перечисляются индексами $a=$ $1, \ldots, N$ и $k=1,2,3$. Здесь и далее принимается соглашение о суммировании в формулах по всем допустимым значениям двукратно повторяющихся индексов.

Важность отдельного изучения случая эволюционных уравнений дивергентного типа связана с тем, что, с физической точки зрения, они обуславливают наличие так называемых локальных законов сохранения для изменяющихся со временем полей $X_{a}(\boldsymbol{x}, t), a=1, \ldots, N$. При этом векторфункции $\left\langle S_{a ; k}, a=1, \ldots, N\right\rangle, k=1,2,3$, играют роль плотностей потоков соответствующих полей.

Таким образом, описание всех дифференциальных операторов дивергентного типа из многообразия $\mathcal{K}_{s}(\mathcal{L})$ состоит в описании линейного многообразия всех дифференциальных операторов вида (2.1), обладающей указанными свойствами ковариантности вектор-функции $S_{a ; k}$. Это многообразие операторов мы будем обозначать тем же символом $\mathcal{K}_{s}(\mathcal{L})$ в тех случаях, когда это не вызовет недоразумений. Справедливо следующее очевидное утверждение.

Теорема 2.1 (см., например, [15]). Для функций

$$
S_{a ; k} \in\left[C_{1, \text { loc }}\left(\mathcal{L} \times\left(\mathbb{R}^{3} \times \mathcal{L}\right) \times \cdots \times\left(\mathbb{R}^{3(s-1)} \times \mathcal{L}\right)\right)\right]^{3 N}
$$


со значениями в $\mathcal{L}^{3}$, которые удовлетворяют условию

$$
\nabla_{k} S_{a ; k}=0
$$

имеет место представление

$$
S_{a ; k}=\varepsilon_{k l m} \nabla_{l} Z_{a ; m}
$$

с некоторой вектор-функиией

$$
Z_{a ; m}(\boldsymbol{X}, \nabla \otimes \boldsymbol{X}, \ldots, \underbrace{\nabla \otimes \cdots \otimes \nabla}_{s} \otimes \boldsymbol{X}) \in\left[C_{1, \mathrm{loc}}\left(\mathcal{L} \times \cdots \times\left(\mathbb{R}^{3(s-2)} \times \mathcal{L}\right)\right)\right]^{3 N},
$$

где $\varepsilon_{k l m}$ - псевдотензор Леви- Чивита в $\mathbb{R}^{3}$.

Следовательно, для решения задачи об описании многообразия $\mathcal{K}_{s}(\mathcal{L})$ необходимо и достаточно описание всех вектор-функций $S_{a ; k}$, удовлетворяющих условию ковариантности при вращении пространства, которые представляют действия дифференциальных операторов $(s-1)$-го порядка, определенных с точностью до произвольной функции $\varepsilon_{k l m} \nabla_{l} Z_{a ; m}$, указанной в лемме.

Далее будем интересоваться многообразием $\mathcal{K}_{2}(\mathcal{L})$ уравнений $(1.1)$ с дифференциальным оператором второго порядка $\mathrm{L}_{a}:\left[C_{2, \text { lос }}\left(\mathbb{R}^{3}\right)\right]^{N} \rightarrow\left[C_{2, \text { lос }}\left(\mathbb{R}^{3}\right)\right]^{N}$. В этом случае функции $S_{a ; k}$ представляются действием квазилинейных дифференциальных операторов первого порядка на поле $\boldsymbol{X}$,

$$
S_{a ; k}(\boldsymbol{X}, \nabla \otimes \boldsymbol{X})=T_{a, b ; k, m}(\boldsymbol{X}) \nabla_{m} X_{b}+U_{a ; k}(\boldsymbol{X}) .
$$

Здесь по индексу $b$ производится суммирование в пределах $b=1, \ldots, N$. Ввиду требования ковариантности набора функций $S_{a ; k}(\boldsymbol{X}, \nabla \otimes \boldsymbol{X})$, коэффициенты $T_{a, b ; k, m}(\boldsymbol{X})$ и функции $U_{a ; k}(\boldsymbol{X})$ являются, соответственно, тензор- и вектор-функциями только от значений полей $X_{a}(\boldsymbol{x}, t)$. (Далее в процессе вычислений в рамках тензорной алгебры мы не делаем различия между ковариантными и контравариантными индексами, что допустимо ввиду евклидовости пространства $\mathbb{R}^{3}$ (см. [13]).)

Следствием формулы (2.2) является то, что для решения задачи о перечислении операторов из $\mathcal{K}_{2}(\mathcal{L})$ необходимо и достаточно описать линейное многообразие всех вектор-функций вида $(2.2)$ с точностью до $\varepsilon_{k l m} \nabla_{l} Z_{a ; m}(\boldsymbol{X})$ с произвольной дважды непрерывно дифференцируемой вектор-функцией $Z_{a ; m}(\boldsymbol{X})$.

Так как функции $T_{a, b ; k, m}(\boldsymbol{X})$ и $U_{a ; k}(\boldsymbol{X})$ не зависят явно от $\boldsymbol{x} \in \mathbb{R}^{3}$, то для описания этого линейного многообразия достаточно описать линейные многообразия всех тензор-функций $T_{a, b ; k, m}(\boldsymbol{X})$ и всех вектор-функций $U_{a ; k}(\boldsymbol{X})$ как функций от вектора $\boldsymbol{X} \in \mathcal{L}$ в пространствах $\left[C_{1, \operatorname{loc}}(\mathcal{L})\right]^{(3 N)^{2}}$ и $\left[C_{1, \operatorname{loc}}(\mathcal{L})\right]^{3 N}$, соответственно.

Заметим, что функции $T_{a, b ; k, m}(\boldsymbol{X})$ и $U_{a ; k}(\boldsymbol{X})$ образуют представления группы $\mathbf{O}_{3}$ соответственно в пространствах представления $\left(\mathcal{L} \times \mathbb{R}^{3}\right)^{2}$ и $\mathcal{L} \times \mathbb{R}^{3}$. Пусть

$$
\left\{T_{a, b ; k, m}^{(\alpha)} ; \alpha \in \mathcal{T}\right\}, \quad\left\{U_{a ; k}(\boldsymbol{X}) ; \beta \in \mathcal{U}\right\}
$$

- конечные наборы функций, составляющих базисы этих представлений. Функции из этих базисов представляют собой линейно независимые мономы относительно тензорного произведения в алгебре с фиксированным набором образующих. Этот набор состоит из неприводимых представлений, составляющих представление $\boldsymbol{X}$, и универсальных для $\mathbb{R}^{3}$ тензора второго ранга $\boldsymbol{\delta}$ (символ Кронекера) и псевдотензора третьего ранга $\varepsilon$ (символ Леви-Чивита).

Используя разложения произвольного представления группы по базису, описание интересующего нас многообразия можем сформулировать следующим образом.

Теорема 2.2. Любые непрерьвно дифференцируемые тензор-функиия $T_{a, b ; k, m}(\boldsymbol{X}): \mathcal{L} \rightarrow(\mathcal{L} \times$ $\left.\mathbb{R}^{3}\right)^{2}$ и вектор-функиия $U_{a ; k}(\boldsymbol{X}): \mathcal{L} \rightarrow \mathcal{L} \times \mathbb{R}^{3}$ определяются бормулами

$$
T_{a, b ; k, m}(\boldsymbol{X})=\sum_{\alpha \in \mathcal{T}} f^{(\alpha)}(\boldsymbol{X}) T_{a, b ; k, m}^{(\alpha)}(\boldsymbol{X}), \quad U_{a ; k}(\boldsymbol{X})=\sum_{\beta \in \mathcal{U}} g^{(\beta)}(\boldsymbol{X}) U_{a ; k}^{(\beta)}(\boldsymbol{X}),
$$


где коэффициенты разложений $f^{(\alpha)}, \alpha \in \mathcal{T}, u g^{(\beta)}, \beta \in \mathcal{U}$, являются непрерывно дифберенцируемыми (скалярными) функииями от совокупности переменных, которая состоит из инвариантов представления $\mathcal{L}$ относительно действия группы $\mathbf{O}_{3}$.

На основе линейных многообразий функций $T_{a, b ; k, m}(\boldsymbol{X}), \alpha \in \mathcal{T}$, и $U_{a ; k}(\boldsymbol{X}), \beta \in \mathcal{U}$, следующее утверждение описывает многообразие $\mathcal{K}_{s}(\mathcal{L})$.

Теорема 2.3. Набор вектор-функций $T_{a, b ; k, m}^{(\alpha)}(\boldsymbol{X}) \nabla_{m} X_{b}, \alpha \in \mathcal{T}$, линейно независим при $\boldsymbol{X} \in$ $\left[C_{2, \text { loc }}\left(\mathbb{R}^{3}\right)\right]^{N}$.

Доказательство. Допустим, что совокупность вектор-функций $T_{a, b ; k, m}^{(\alpha)}(\boldsymbol{X}) \nabla_{m} X_{b}, \alpha \in \mathcal{T}$, линейно зависима, т.е. найдутся такие коэффициенты $c^{(\alpha)}, \alpha \in \mathcal{T}$, что тождественно выполняется равенство

$$
\sum_{\alpha \in \mathcal{T}} c^{(\alpha)} T_{a, b ; k, m}^{(\alpha)}(\boldsymbol{X}) \nabla_{m} X_{b}=0 .
$$

Ввиду произвольности поля $\boldsymbol{X}$, линеаризуем это уравнение около функции $\boldsymbol{X}=$ const:

$$
\sum_{\alpha \in \mathcal{T}} c^{(\alpha)} T_{a, b ; k, m}^{(\alpha)}(\boldsymbol{X}) \nabla_{m} \delta X_{b}=0
$$

Положим в этом равенстве $\delta X_{b}=A_{b} \exp (\boldsymbol{k}, \boldsymbol{x})$ с произвольными постоянными набором $A_{b}$, $b=1, \ldots, N$, и вектором $\boldsymbol{k} \in \mathbb{R}^{3}$; здесь и далее $(\cdot, \cdot)$ обозначает скалярное произведение векторов из $\mathbb{R}^{3}$. В результате получим равенство

$$
\sum_{\alpha \in \mathcal{T}} c^{(\alpha)} T_{a, b ; k, m}^{(\alpha)}(\boldsymbol{X}) A_{b} k_{m}=0 .
$$

Ввиду произвольности векторов $\boldsymbol{k} \in \mathbb{R}^{3}$ и $\boldsymbol{A}=\left\langle A_{b}, b=1, \ldots, N\right\rangle \in \mathcal{L}$, продифференцируем его по компонентам этих векторов. В результате находим, что имеет место тождество

$$
\sum_{\alpha \in \mathcal{T}} c^{(\alpha)} T_{a, b ; k, m}^{(\alpha)}(\boldsymbol{X})=0
$$

Это указывает на линейную зависимость базисных функций линейного представления, представленного тензор-функциями $T_{a, b ; k, m}^{(\alpha)}(\boldsymbol{X})$.

Следствие 2.1. Все вектор-функиии $S_{a ; k}(\boldsymbol{X}, \nabla \otimes \boldsymbol{X})$, определяющие элементв линейного многообразия $\mathcal{K}_{s}(\mathcal{L})$, представляются формулой

$$
S_{a ; k}(\boldsymbol{X}, \nabla \otimes \boldsymbol{X})=\sum_{\alpha \in \mathcal{T}} f^{(\alpha)}(\boldsymbol{X}) T_{a, b ; k, m}^{(\alpha)}(\boldsymbol{X}) \nabla_{m} X_{b}+\sum_{\beta \in \mathcal{U}} g^{(\beta)}(\boldsymbol{X}) U_{a ; k}^{(\beta)}(\boldsymbol{X}),
$$

с точностью до функиии $\varepsilon_{k l m} \nabla_{l} Z_{a ; m}, k=1,2,3$, с произвольной дважды непрерывно дифберениируемой вектор-функиией $Z_{a ; m}(\boldsymbol{X}), a=1, \ldots, N, m=1,2,3$.

Так как при всех наборах произвольно выбранных коэффициентных функций $f^{(\alpha)}(\boldsymbol{X}), \alpha \in \mathcal{T}$, и $g^{(\beta)}(\boldsymbol{X}), \beta \in \mathcal{U}$, выражение для функции $S_{a ; k}(\boldsymbol{X}, \nabla \otimes \boldsymbol{X}), k=1,2,3$, даваемое формулой $(2.3)$, не может стать равным $\varepsilon_{k l m} \nabla_{l} Z_{a ; m}$ с некоторой вектор-функцией $Z_{a ; m}(\boldsymbol{X}), m=1,2,3, a=1, \ldots, N$, то справедливо следующее утверждение.

Следствие 2.2. Полное описание линейного многообразия $\mathcal{K}_{s}(\mathcal{L})$ дифференциальных операторов $\mathrm{L}_{a}[\boldsymbol{X}]$ дается формулой (2.1), где вектор-функиии $S_{a ; k}(\boldsymbol{X}, \nabla \otimes \boldsymbol{X})$ определяются формулой (2.3).

Таким образом, задача описания многообразия $\mathcal{K}_{s}(\mathcal{L})$ сводится к задаче об описании набора базисных функций $T_{a, b ; k, m}^{(\alpha)}, \alpha \in \mathcal{T}$, и $U_{a ; k}^{(\beta)}, \beta \in \mathcal{U}$, соответствующих представлений группы $\mathbf{O}_{3}$, набор аргументов которых состоит из набора $\boldsymbol{X}$ полей из пространства представления $\mathcal{L}$ и которые ковариантны в соответствии с преобразованиями группы $\mathbf{O}_{3}$. 
3. Описание многообразия $\mathcal{K}_{2}\left(\mathbb{R}^{3}\right)$ для векторного поля. На основании общей формулировки задачи, данной в предыдущем разделе, сформулируем теперь конкретную задачу об описании многообразия $\mathcal{K}_{2}(\mathcal{L})$ всех эволюционных уравнений для векторного поля. В этом случае набор $\boldsymbol{X}$ состоит из компонент вектора $\boldsymbol{P}=\left\langle P_{j} ; j=1,2,3\right\rangle$, т.е. $\mathcal{L}=\mathbb{R}^{3}$.

Вводя соответствующие переобозначения для компонент плотности потока $S_{j ; k}(\boldsymbol{P}, \nabla \otimes \boldsymbol{P})$ и коэффициентов $U_{j ; k}^{(\beta)}(\boldsymbol{P}), T_{j, l ; k, m}(\boldsymbol{P})$ разложения $(2.2)$, запишем его в виде

$$
S_{j ; k}(\boldsymbol{P}, \nabla \otimes \boldsymbol{P})=T_{j, l ; k, m}(\boldsymbol{P}) \nabla_{m} P_{l}+U_{j ; k}(\boldsymbol{P}) .
$$

Применение элементов группы $\mathbf{O}_{3}$ к левой части равенства преобразует ее как тензор второго ранга. По этой причине коэффициенты $T_{j, l ; k, m}(\boldsymbol{P})$ и $U_{j ; k}(\boldsymbol{P})$ являются соответственно тензорами четвертого и второго ранга. Таким образом, решение задачи о перечислении всех дифференциальных операторов из $\mathcal{K}_{2}\left(\mathbb{R}^{3}\right)$, согласно следствию 2.2 , состоит в определении наборов $\mathcal{T}$ и $\mathcal{U}$, соответственно, всех базисных функций для представлений группы $\mathbf{O}_{3}$ в виде тензоров четвертого и второго ранга. Искомые базисные функции будем искать в виде мономов относительно тензорного произведения. Набор образующих тензорной алгебры в сферически симметричном случае состоит из фундаментальных тензора второго ранга $\boldsymbol{\delta}$ и псевдотензора третьего ранга $\boldsymbol{\varepsilon}$, а также вектора $\boldsymbol{P}$.

Технически решение соответствующей алгебраической задачи не вызывает затруднений. Полученный результат сформулируем в следующем виде.

Теорема 3.1 (см. [14]). Набор $\mathcal{U}$ состоит из двух тензоров $\boldsymbol{\delta}$ и $\boldsymbol{P} \otimes \boldsymbol{P}$, а набор $\mathcal{T}$ состоит из 10 элементов следующих типов:

(1) 3 тензора вида $\boldsymbol{\delta} \otimes \boldsymbol{\delta}$;

(2) 6 тензоров $\boldsymbol{P} \otimes \boldsymbol{P} \otimes \boldsymbol{\delta}$;

(3) тензор $\boldsymbol{P} \otimes \boldsymbol{P} \otimes \boldsymbol{P} \otimes \boldsymbol{P}$.

Доказательство. Доказательство проводится перебором всех имеющихся возможностей конструирования мономов из образующих так, чтобы получаемые мономы приводили к тензорному представлению требуемого типа.

Проверка линейной независимости всех получившихся мономов $T_{j, l ; k, m}^{(\alpha)}(\boldsymbol{P})$ сводится к проверке линейной независимости мономов отдельно в каждой из групп, указанных в формулировке теоремы, так как элементы разных групп имеют различные степени вхождения в их состав произвольного вектора $\boldsymbol{P}$ : в элементы первой группы он не входит, в элементы второй группы он входит квадратично, а в единственный элемент третьей группы он входит в четвертой степени. Тогда элемент $\boldsymbol{P} \otimes \boldsymbol{P} \otimes \boldsymbol{P} \otimes \boldsymbol{P}$ линейно независим от остальных. Проверка линейной независимости элементов в первой и второй группах представляет анализ уравнений для коэффициентов $c^{(\alpha)}, \alpha=1, \ldots, 9$ :

для первой группы,

$$
c^{(1)} T_{j, l ; k, m}^{(1)}(\boldsymbol{P})+c^{(2)} T_{j, l ; k, m}^{(2)}(\boldsymbol{P})+c^{(3)} T_{j, l ; k, m}^{(3)}(\boldsymbol{P})=0
$$

$$
\sum_{\alpha=4}^{9} c^{(\alpha)} T_{j, l ; k, m}^{(\alpha)}(\boldsymbol{P})=0
$$

для второй группы. В свою очередь, доказательство равенств $c^{(\alpha)}=0, \alpha=1, \ldots, 9$, сводится к проверке на невырожденность двух простых систем однородных линейных уравнений для коэффициентов для коэффициентов $c^{(\alpha)}, \alpha=1,2,3$, и $c^{(\alpha)}, \alpha=4, \ldots, 9$, соответственно.

Так как в сферически симметричном случае имеется только один инвариант $\boldsymbol{P}^{2}$ группы вращений, то, используя общую формулу (2.2) для случая векторного поля, получаем решение задачи об описании всех плотностей потоков поля, определяющих все многообразие $\mathcal{K}_{2}\left(\mathbb{R}^{3}\right)$ дифференциальных операторов дивергентного типа, действующих в пространстве дважды непрерывно дифференцируемых векторных полей $\boldsymbol{P}$ на $\mathbb{R}^{3}$,

$$
S_{j ; k}(\boldsymbol{P}, \nabla \otimes \boldsymbol{P})=g^{(1)}\left(\boldsymbol{P}^{2}\right) \delta_{j k}+g^{(2)}\left(\boldsymbol{P}^{2}\right) P_{j} P_{k}+\sum_{\alpha=1}^{10} f^{(\alpha)}\left(\boldsymbol{P}^{2}\right) T_{j, l ; k, m}^{(\alpha)}(\boldsymbol{P}) \nabla_{m} P_{l},
$$


где произведено переобозначение произвольных коэффициентных функций

$$
f^{(\alpha)}(\boldsymbol{P}) \Rightarrow f^{(\alpha)}\left(\boldsymbol{P}^{2}\right), \quad \alpha=1, \ldots, 10, \quad g^{(\beta)}(\boldsymbol{P}) \Rightarrow g^{(\beta)}\left(\boldsymbol{P}^{2}\right), \quad \beta=1,2,
$$

на непрерывно дифференцируемые функции на $(0, \infty)$, зависящие от $\boldsymbol{P}^{2}$. Суммируемые с произвольными коэффициентами $f^{(\alpha)}\left(\boldsymbol{P}^{2}\right)$ свертки $T_{j, l ; k, m}^{(\alpha)}(\boldsymbol{P}) \nabla_{m} P_{l}$ в представленной формуле (3.1) даются в обозначениях алгебры векторов в $\mathbb{R}^{3}$ следующим списком, составленным согласно порядку, который указан в формулировке теоремы:

$$
\begin{gathered}
\delta_{j k}(\nabla, \boldsymbol{P}), \quad \nabla_{j} P_{k} \pm \nabla_{k} P_{j} ; \\
\delta_{j k}(\boldsymbol{P}, \nabla) \boldsymbol{P}^{2}, \quad P_{k} \nabla_{j} \boldsymbol{P}^{2} \pm P_{j} \nabla_{k} \boldsymbol{P}^{2}, \quad P_{j}(\boldsymbol{P}, \nabla) P_{k} \pm P_{k}(\boldsymbol{P}, \nabla) P_{j}, \\
P_{j} P_{k}(\boldsymbol{P}, \nabla) \boldsymbol{P}^{2} .
\end{gathered}
$$

Здесь мы представили плотности потоков, образовав из них симметричные и антисимметричные комбинации, имея в виду их неприводимость.

Таким образом, результатом рассмотрений этого раздела является следующая теорема.

Теорема 3.2 (см. [14]). Все дифференциальные операторы $\mathrm{L}_{j}[\boldsymbol{P}]$ из $\mathcal{K}_{2}\left(\mathbb{R}^{3}\right)$ дивергентного muna

$$
\mathrm{L}_{j}[\boldsymbol{P}]=\nabla_{k} S_{j ; k}(\boldsymbol{P}, \nabla \otimes \boldsymbol{P})
$$

на пространстве дважсды непрерывно дифферениируемых векторных полей $\boldsymbol{P}$ на $\mathbb{R}^{3}$ определяются формулой (3.1), в которой $g^{(1)}, g^{(2)}, f^{(\alpha)}, \alpha=1, \ldots, 10,-$ произволъные непрерывно дифферениируемые функиии от $\boldsymbol{P}^{2}$ и тензоры $T_{j, l ; k, m}^{(\alpha)}(\boldsymbol{P})$ определяются формулами (3.2)-(3.4).

4. Описание многообразия $\mathcal{K}_{2}\left(\mathbb{R}^{3}\right)$ для псевдовекторного поля. Рассмотрим теперь многообразие $\mathcal{K}_{2}\left(\mathbb{R}^{3}\right)$ всех дифференциальных операторов второго порядка дивергентного типа для псевдовекторного поля. В этом случае набор $\boldsymbol{X}$ состоит из компонент псевдовектора $\boldsymbol{M}=\left\langle M_{j} ; j=1,2,3\right\rangle$ в пространстве $\mathcal{L}=\mathbb{R}^{3}$. Вводя переобозначения для компонент плотности потока $S_{j ; k}(\boldsymbol{M}, \nabla \otimes \boldsymbol{M})$, аналогичные тем, которые были использованы в разделе 3 , и для функций $U_{j ; k}^{(\beta)}(\boldsymbol{M}), T_{j, l ; k, m}(\boldsymbol{M})$ в формуле $(2.2)$ запишем ее в виде

$$
S_{j ; k}(\boldsymbol{M}, \nabla \otimes \boldsymbol{M})=T_{j, l ; k, m}(\boldsymbol{M}) \nabla_{m} X_{l}+U_{j ; k}(\boldsymbol{M}) .
$$

Так как применение элементов группы $\mathbf{O}_{3}$ к левой части равенства преобразует ее как тензор второго ранга в случае непрерывных поворотов пространства и изменяет знак на обратный при отражении $\mathbb{R}^{3}$, то $S_{j ; k}(\boldsymbol{M}, \nabla \otimes \boldsymbol{M})$ представляет собой псевдотензор второго ранга. По этой причине коэффициенты $T_{j, l ; k, m}(\boldsymbol{M})$ и $U_{j ; k}(\boldsymbol{M})$ являются, соответственно, тензором четвертого ранга и псевдотензором второго ранга.

Таким образом, решение задачи о перечислении операторов из $\mathcal{K}_{2}(\mathcal{L})$ состоит в определении наборов $\mathcal{T}$ и $\mathcal{U}$ всех линейно независимых мономов относительно тензорного произведения, представляющих, соответственно, тензоры четвертого ранга и псевдотензоры второго ранга. Как и в предыдущем разделе, исследуем сферически симметричный случай, когда образующими тензорной алгебры являются тензор $\boldsymbol{\delta}$ и псевдотензор $\boldsymbol{\varepsilon}$, а также псевдовектор $\boldsymbol{M}$. В результате несложного алгебраического анализа приходим к следующему утверждению.

Теорема 4.1 (см. [4]). Набор $\mathcal{U}$ не содержит ни одного элемента, а набор $\mathcal{T}$ состоит из 26 элементов, представленных следующим списком:

(1) 3 тензора вида $\boldsymbol{\delta} \otimes \boldsymbol{\delta}$

(2) 6 тензоров $\boldsymbol{M} \otimes \boldsymbol{M} \otimes \boldsymbol{\delta}$;

(3) 6 тензоров $\boldsymbol{\delta} \otimes\left(\boldsymbol{M}^{\wedge} \boldsymbol{\varepsilon}\right)$;

(4) 4 тензора $\boldsymbol{M} \otimes \boldsymbol{\varepsilon}$;

(5) 6 тензоров $\boldsymbol{M} \otimes \boldsymbol{M} \otimes\left(\boldsymbol{M}^{\wedge} \boldsymbol{\varepsilon}\right)$;

(6) тензор $\boldsymbol{M} \otimes \boldsymbol{M} \otimes \boldsymbol{M} \otimes \boldsymbol{M}$. 
Здесь знак - - символ операции свертки.

Доказательство. Доказательство основано на том, что при конструировании мономов в виде тензоров четвертого ранга псевдовектор $\boldsymbol{M}$ и псевдотензор $\boldsymbol{\varepsilon}$ должны входить в состав тензорного произведения суммарно четное число раз. При этом символ Леви-Чивита может появляться не более одного раза ввиду тензорного тождества

$$
\varepsilon_{i j k} \varepsilon_{l m n}=\operatorname{det}\left(\begin{array}{ccc}
\delta_{i l} & \delta_{i m} & \delta_{i n} \\
\delta_{j l} & \delta_{j m} & \delta_{j n} \\
\delta_{k l} & \delta_{k m} & \delta_{k n}
\end{array}\right),
$$

(см., например, [12]), которое выражает тензорное произведение символов через линейную комбинацию мономов, в которые символ Леви-Чивита не входит.

Проверка линейной независимости всех получившихся мономов $T_{j, l ; k, m}^{(\alpha)}(\boldsymbol{M})$ сводится к проверке их линейной независимости отдельно в каждой из следующих групп из числа указанных в формулировке теоремы. Эти группы объединяют все те мономы, которые имеют одинаковые степени вхождения в их состав псевдовектора $\boldsymbol{M}$, а именно, в элементы первой группы он не входит, в элементы третьей и четвертой групп он входит линейно, в элементы второй группы он входит квадратично, степень его вхождения в элементы пятой группы равна 3 и, наконец, эта степень равна 4 для единственного элемента шестой группы. Элемент $\boldsymbol{M} \otimes \boldsymbol{M} \otimes \boldsymbol{M} \otimes \boldsymbol{M}$ линейно независим от остальных. Проверка линейной независимости элементов во всех остальных группах представляет собой анализ уравнений для коэффициентов $c^{(\alpha)}, \alpha=1, \ldots, 25$, которые перечислены согласно указанному порядку:

$$
\begin{gathered}
c^{(1)} T_{j, l ; k, m}^{(1)}(\boldsymbol{M})+c^{(2)} T_{j, l ; k, m}^{(2)}(\boldsymbol{M})+c^{(3)} T_{j, l ; k, m}^{(3)}(\boldsymbol{M})=0, \\
\sum_{\alpha=10}^{19} c^{(\alpha)} T_{j, l ; k, m}^{(\alpha)}(\boldsymbol{M})=0, \quad \sum_{\alpha=4}^{9} c^{(\alpha)} T_{j, l ; k, m}^{(\alpha)}(\boldsymbol{M})=0, \quad \sum_{\alpha=20}^{25} c^{(\alpha)} T_{j, l ; k, m}^{(\alpha)}(\boldsymbol{M})=0 .
\end{gathered}
$$

Анализ этих уравнений сводится к проверке на невырожденность не связанных друг с другом систем однородных линейных уравнений для коэффициентов для коэффициентов $c^{(\alpha)}, \alpha=1, \ldots, 25$, имеющихся в указанных уравнениях, из которых первые две системы состоят соответственно из 3 и 10 уравнений, а вторые две содержат по 6 уравнений каждая.

Так как в рассматриваемом сферически симметричном случае имеется только один инвариант $\boldsymbol{M}^{2}$ группы вращений, то, используя общее представление (2.2) для случая псевдовекторного поля, получаем решение задачи об описании всех плотностей потоков псевдовекторного поля, определяющих все могообразие $\mathcal{K}_{2}\left(\mathbb{R}^{3}\right)$ дифференциальных операторов дивергентного типа в пространстве дважды непрерывно дифференцируемых псевдовекторных полей $\boldsymbol{M}$ на $\mathbb{R}^{3}$,

$$
S_{j ; k}(\boldsymbol{M}, \nabla \otimes \boldsymbol{M})=\sum_{\alpha=1}^{26} f^{(\alpha)}\left(\boldsymbol{M}^{2}\right) T_{j, l ; k, m}^{(\alpha)}(\boldsymbol{M}) \nabla_{m} M_{l},
$$

где произведено переобозначение произвольных коэффициентных функций $f^{(\alpha)}(\boldsymbol{M}) \Rightarrow f^{(\alpha)}\left(\boldsymbol{M}^{2}\right)$, $\alpha=1, \ldots, 26$, на непрерывно дифференцируемые функции на $(0, \infty)$, зависящие от единственного инварианта $\boldsymbol{M}^{2}$.

Свертки $T_{j, l ; k, m}^{(\alpha)}(\boldsymbol{M}) \nabla_{m} M_{l}$ в формуле (4.1) представляют собой квазилинейные дифференциальные операторы первого порядка. Они даются в обозначениях алгебры векторов в $\mathbb{R}^{3}$ следующим списком, составленным согласно порядку, который указан в формулировке теоремы:

$$
\begin{aligned}
& \delta_{j k}(\nabla, \boldsymbol{M}), \quad \nabla_{j} M_{k} \pm \nabla_{k} M_{j} ; \\
& \delta_{j k}(\boldsymbol{M}, \nabla) \boldsymbol{M}^{2}, \quad M_{k} \nabla_{j} \boldsymbol{M}^{2} \pm M_{j} \nabla_{k} \boldsymbol{M}^{2}, \quad M_{j}(\boldsymbol{M}, \nabla) M_{k} \pm M_{k}(\boldsymbol{M}, \nabla) M_{j}, \quad M_{j} M_{k}(\nabla, \boldsymbol{M}) ; \\
& \delta_{j k}(\boldsymbol{M},[\nabla, \boldsymbol{M}]),[\boldsymbol{M}, \nabla]_{j} M_{k} \pm[\boldsymbol{M}, \nabla]_{k} M_{j}, \varepsilon_{j l n} M_{n} \nabla_{k} M_{l} \pm \varepsilon_{k l n} M_{n} \nabla_{j} M_{l}, \varepsilon_{j k m} M_{m}(\nabla, \boldsymbol{M}) ; \\
& M_{j}[\nabla, \boldsymbol{M}]_{k} \pm M_{k}[\nabla, \boldsymbol{M}]_{j}, \quad \varepsilon_{j k l}(\boldsymbol{M}, \nabla) M_{l}, \quad \varepsilon_{j k l} \nabla_{l} \boldsymbol{M}^{2} \\
& M_{j} M_{k}(\boldsymbol{M},[\nabla, \boldsymbol{M}]), \quad \varepsilon_{j k m} M_{m}(\boldsymbol{M}, \nabla) \boldsymbol{M}^{2},
\end{aligned}
$$




$$
\begin{gathered}
M_{j}[\boldsymbol{M},(\boldsymbol{M}, \nabla) \boldsymbol{M}]_{k} \pm M_{k}[\boldsymbol{M},(\boldsymbol{M}, \nabla) \boldsymbol{M}]_{j}, \quad M_{j}[\boldsymbol{M}, \nabla]_{k} \boldsymbol{M}^{2} \pm M_{k}[\boldsymbol{M}, \nabla]_{j} \boldsymbol{M}^{2} \\
M_{j} M_{k}(\boldsymbol{M}, \nabla) \mathbf{M}^{2} .
\end{gathered}
$$

Как и при изучении случая векторного поля, мы представили мономы, образовав из них симметричные и антисимметричные комбинации, ввиду их линейной независимости.

На основе суммы (4.1) всех указанных линейных дифференциальных операторов с произвольными коэффициентами $f^{(\alpha)}\left(\boldsymbol{M}^{2}\right)$ получаем в результате наших рассмотрений в этом разделе следующее утверждение.

Теорема 4.2. Все операторы $\mathrm{L}_{j}[\boldsymbol{M}] \in \mathcal{K}_{2}(\mathcal{L})$ в случае псевдовекторного поля $\boldsymbol{M}$ представляются следующей формулой:

$$
\mathrm{L}_{j}[\boldsymbol{M}]=\nabla_{k} S_{j ; k}(\boldsymbol{M}, \nabla \otimes \boldsymbol{M})=\sum_{\alpha=1}^{26} \nabla_{k} f^{(\alpha)}\left(\boldsymbol{M}^{2}\right) T_{j, l ; k, m}^{(\alpha)}(\boldsymbol{M}) \nabla_{m} M_{l},
$$

где операторь первого порядка $T_{j, l ; k, m}^{(\alpha)}(\boldsymbol{M}) \nabla_{m} M_{l}, \alpha=1, \ldots, 26$, представлены списком (4.2)(4.8).

5. Класс $\mathcal{K}_{2}\left(\mathbb{R}^{3}\right) \cap \mathcal{M}_{0}\left(\mathbb{R}^{3}\right)$ для векторного поля. В этом и следующем разделах представлены основные и довольно неочевидные результаты работы. Мы дадим описание многообразий, которые состоят из всех тех операторов $\mathrm{L}_{j}$ из $\mathcal{K}_{2}\left(\mathbb{R}^{3}\right)$, которые принадлежат классу операторов $\mathcal{M}_{0}\left(\mathbb{R}^{3}\right)$, сохраняющих свойства унимодальности и соленоидальности в процессе эволюции поля $\boldsymbol{M}$. Требование сохранения указанных свойств поля представляет собой как раз те специальные ограничения при конструировании подходящего оператора, адекватного физическим условиям задачи. В этом разделе мы покажем, что класс $\mathcal{K}_{2}\left(\mathbb{R}^{3}\right) \cap \mathcal{M}_{0}\left(\mathbb{R}^{3}\right)$ тривиален, т.е.состоит только из нулевого оператора.

Пусть поле $\boldsymbol{P}(\boldsymbol{x}, t)$ обладает свойством унимодальности, $\boldsymbol{P}^{2}(\boldsymbol{x}, t)=P^{2}=$ const. Тогда все функции $f^{(\alpha)}\left(\boldsymbol{P}^{2}\right), \alpha=1, \ldots, 10$, и $g^{(\beta)}\left(\boldsymbol{P}^{2}\right), \beta=1,2$, являются постоянными. Более того, при этом условии плотности потоков $S_{j ; k}$, даваемые выражениями $(3.3)$ и $(3.4)$, у которых имеются производные $\nabla_{j} \boldsymbol{P}^{2}$, обращаются в нуль. Кроме того, если поле $\boldsymbol{P}(\boldsymbol{x}, t)$ соленоидально, $(\nabla, \boldsymbol{P})=0$, то обращаются в нуль плотности потоков в выражениях (3.2), (3.3), в которых присутствует его дивергенция. В результате при выполнении условий унимодальности и соленоидальности поля $\boldsymbol{P}$, общее уравнение $\dot{P}_{j}=\mathrm{L}_{j}[\boldsymbol{P}]$, описывающее его динамику, с дифференциальным оператором, который описывается теоремой 3.2 и формулой (3.1), принимает вид

$$
\begin{aligned}
\dot{P}_{j}=g^{(2)}(\boldsymbol{P}, \nabla) & P_{j}+\left(f^{(2)}-f^{(3)}\right) \Delta P_{j}+ \\
& +f^{(7)} \nabla_{k}\left(P_{j}(\boldsymbol{P}, \nabla) P_{k}+P_{k}(\boldsymbol{P}, \nabla) P_{j}\right)+f^{(8)} \nabla_{k}\left(P_{j}(\boldsymbol{P}, \nabla) P_{k}-P_{k}(\boldsymbol{P}, \nabla) P_{j}\right) .
\end{aligned}
$$

Здесь использована нумерация постоянных $f^{(\alpha)}$ согласно порядку соответствующих им плотностей потоков, описанных формулами (3.2)-(3.4).

Для того чтобы оператор $L_{j}[\boldsymbol{P}]$ сохранял свойства унимодальности и соленоидальности поля $\boldsymbol{P}(\boldsymbol{x}, t)$, необходимо и достаточно, чтобы для любого дважды непрерывно дифференцируемого поля на $\mathbb{R}^{3}$ тождественно выполнялись равенства

$$
\nabla_{j} \mathrm{~L}_{j}[\boldsymbol{P}]=0, \quad P_{j} \mathrm{~L}_{j}[\boldsymbol{P}]=0 .
$$

Теорема 5.1. $\mathcal{K}_{2}\left(\mathbb{R}^{3}\right) \cap \mathcal{M}_{0}\left(\mathbb{R}^{3}\right)=\{0\}$.

Доказательство. Покажем, что если поле $\boldsymbol{P}$ обладает свойствами $\boldsymbol{P}^{2}=P^{2},(\nabla, \boldsymbol{P})=0$, то равенства (5.2) могут быть удовлетворены только в случае, когда $f^{(3)}=f^{(2)}, f^{(7)}=f^{(8)}=0$.

Умножим скалярно правую часть уравнения (5.2) на $\boldsymbol{P}$. Так как $(\boldsymbol{P},(\boldsymbol{P}, \nabla) \boldsymbol{P})=(\nabla, \boldsymbol{P}) \boldsymbol{P}^{2}=0$, то, в предположении о выполнимости соотношения $(\boldsymbol{P}, \mathrm{L}[(\boldsymbol{P})])=0$, получаем равенство

$$
\begin{aligned}
\left(f^{(2)}-\right. & \left.f^{(3)}\right) P_{j} \Delta P_{j}+ \\
& +f^{(7)} P_{j} \nabla_{k}\left(P_{j}(\boldsymbol{P}, \nabla) P_{k}+P_{k}(\boldsymbol{P}, \nabla) P_{j}\right)+f^{(8)} P_{j} \nabla_{k}\left(P_{j}(\boldsymbol{P}, \nabla) P_{k}-P_{k}(\boldsymbol{P}, \nabla) P_{j}\right)=0 .
\end{aligned}
$$


Так как, по предположению, $\boldsymbol{P}^{2}=\mathrm{const}$, то $P_{j} \nabla_{k} P_{j}=0$ и $\nabla_{k} P_{k}=0$; поэтому

$$
P_{j} \nabla_{k} P_{j}(\boldsymbol{P}, \nabla) P_{k}=P^{2} \nabla_{k} P_{l} \nabla_{l} P_{k}+\left(P_{j} \nabla_{k} P_{j}\right) \cdot(\boldsymbol{P}, \nabla) P_{k}=P^{2} \mathrm{Sp} \mathrm{A}^{2},
$$

где введена матрица $A_{k l}=(\mathrm{A})_{k l} \equiv \nabla_{k} P_{l}$. Точно так же получаем, что

$$
P_{j} \nabla_{k} P_{k}(\boldsymbol{P}, \nabla) P_{j}=\nabla_{k} P_{k} P_{j}(\boldsymbol{P}, \nabla) P_{j}-\left((\boldsymbol{P}, \nabla) P_{j}\right) \cdot\left((\boldsymbol{P}, \nabla) P_{j}\right)=-\left(P_{l} A_{l j}\right)^{2} .
$$

Наконец,

$$
P_{j} \Delta P_{j}=P_{j} \nabla_{k} \nabla_{k} P_{j}=\nabla_{k}\left(P_{j} \nabla_{k} P_{j}\right)-\left(\nabla_{k} P_{j}\right) \cdot\left(\nabla_{k} P_{j}\right)=-\mathrm{SpAA}^{T} .
$$

Таким образом, подставляя полученные результирующие выражения (5.4)-(5.6) в (5.3), получаем

$$
c^{(1)} \mathrm{Sp}\left(\mathrm{AA}^{T}\right)+c^{(2)} \mathrm{Sp}^{2}+c^{(3)}\left(P_{l} A_{l j}\right)^{2}=0,
$$

где

$$
c^{(1)}=f^{(3)}-f^{(2)}, \quad c^{(2)}=\left(f^{(7)}+f^{(8)}\right) P^{2}, \quad c^{(3)}=f^{(8)}-f^{(7)} .
$$

Подставим в (5.7) разложение $P_{j}=P_{j}^{(0)}+A_{j k}^{(0)} x_{k}+\ldots$, где $\boldsymbol{P}^{(0)}=\boldsymbol{P}(0)$ - постоянный вектор и $A_{j k}^{(0)}$ - матричные элементы в пространственной точке $\boldsymbol{x}=0$. Рассмотрим член нулевой степени по $\boldsymbol{x}$ равенства (5.7), получившегося в результате разложения левой части. При этом на матричные элементы $A_{j k}^{(0)}$ наложены два условия: $A_{j j}^{(0)}=0$, в силу $(\nabla, \boldsymbol{P})_{0}=0$, и вектор $P_{j}^{(0)}$ является ее левым собственным вектором с нулевым собственным значением. В остальном эта матрица произвольна. Подставляя последовательно в равенство (5.7) матрицы А

$$
\left(\begin{array}{lll}
0 & 1 & 0 \\
0 & 0 & 0 \\
0 & 0 & 0
\end{array}\right), \quad\left(\begin{array}{ccc}
1 & 0 & 0 \\
0 & -1 & 0 \\
0 & 0 & 0
\end{array}\right), \quad\left(\begin{array}{ccc}
1 & 0 & 0 \\
0 & -1 & 0 \\
1 & 1 & 0
\end{array}\right)
$$

с вектором $P_{l}^{(0)}=\langle 0,0,1\rangle$, находим в соответствии с порядком подстановки, что $c_{1}=0, c_{2}=0$, $c_{3}=0$, т.е. $f^{(2)}=f^{(3)}, f^{(7)}=f^{(8)}=0$.

После подстановки этих равенств в (5.1), нужно проверить, может ли уравнение

$$
\dot{P}_{j}=g^{(2)}(\boldsymbol{P}, \nabla) P_{j}, \quad j=1,2,3,
$$

обладать инвариантом $(\nabla, \boldsymbol{P})=0$ с непрерывно дифференцируемым полем $\boldsymbol{P}$ на $\mathbb{R}^{3}$ при условии, что $\boldsymbol{P}^{2}=$ const. Допустив такую возможность и применив операцию дивергенции к обеим частям уравнения, находим

$$
0=\nabla_{j}(\boldsymbol{P}, \nabla) P_{j}=(\boldsymbol{P}, \nabla) \boldsymbol{P}+\left(\nabla_{j} P_{l}\right) \cdot\left(\nabla_{l} P_{j}\right)=\mathrm{SpA}^{2},
$$

что в общем случае невозможно для матриц А с указанными выше свойствами. Достаточно положить ее симметричной.

6. Описание класса $\mathcal{K}_{2}\left(\mathbb{R}^{3}\right) \cap \mathcal{M}_{0}\left(\mathbb{R}^{3}\right)$ для псевдовекторного поля. Пусть псевдовекторное поле $\boldsymbol{M}(\boldsymbol{x}, t)$ обладает свойством унимодальности, $\boldsymbol{M}^{2}(\boldsymbol{x}, t)=M^{2}=$ const. Все функции $f^{(\alpha)}\left(\boldsymbol{M}^{2}\right), \alpha=1, \ldots, 26$, превращаются в постоянные. При этом условии плотности потоков $S_{j ; k}$, даваемые выражениями (4.3) и (4.5)-(4.8), у которых имеются производные $\nabla_{j} \boldsymbol{M}^{2}$, обращаются в нуль. Таковыми являются плотности с номерами 4-6, 19, 21, 24-26, где их нумерация соответствует порядку, представленному в приведенном списке. Если, дополнительно, поле $\boldsymbol{M}(\boldsymbol{x}, t)$ соленоидально, $(\nabla, \boldsymbol{M})=0$, то обращаются в нуль плотности потоков с номерами 1,9 , 15 , в которых присутствует дивергенция $(\nabla, \boldsymbol{M})$.

В результате при выполнении условий унимодальности и соленоидальности поля $\boldsymbol{M}$ общее уравнение $\dot{M}_{j}=\mathrm{L}_{j}[\boldsymbol{M}]$, описывающее его динамику, с дифференциальным оператором, который дается утверждением теоремы 4.2 и формулой (4.1), принимает вид

$$
\begin{gathered}
\dot{M}_{j}=\left(f^{(2)}-f^{(3)}\right) \Delta M_{j}+\left(f^{(7)}+f^{(8)}\right) \nabla_{k}\left(M_{j}(\boldsymbol{M}, \nabla) M_{k}\right)+\left(f^{(7)}-f^{(8)}\right) \nabla_{k}\left(M_{k}(\boldsymbol{M}, \nabla) M_{j}\right)+ \\
+f^{(10)} \nabla_{j}(\boldsymbol{M},[\nabla, \boldsymbol{M}])+\left(f^{(11)}+f^{(12)}\right) \nabla_{k}\left([\boldsymbol{M}, \nabla]_{j} M_{k}\right)+\left(f^{(11)}-f^{(12)}\right) \nabla_{k}\left([\boldsymbol{M}, \nabla]_{k} M_{j}\right)+ \\
+\varepsilon_{j l n}\left(f^{(13)}+f^{(14)}\right) \nabla_{k}\left(M_{n} \nabla_{k} M_{l}\right)+\varepsilon_{k l n}\left(f^{(13)}-f^{(14)}\right) \nabla_{k}\left(M_{n} \nabla_{j} M_{l}\right)+
\end{gathered}
$$




$$
\begin{gathered}
+\left(f^{(16)}+f^{(17)}\right) \nabla_{k}\left(M_{j}[\nabla, \boldsymbol{M}]_{k}\right)+\left(f^{(16)}-f^{(17)}\right) \nabla_{k}\left(M_{k}[\nabla, \boldsymbol{M}]_{j}\right)+ \\
+\varepsilon_{j k l} f^{(18)} \nabla_{k}(\boldsymbol{M}, \nabla) M_{l}+f^{(20)} \nabla_{k} M_{j} M_{k}(\boldsymbol{M},[\nabla, \boldsymbol{M}])+ \\
+\left(f^{(22)}+f^{(23)}\right) \nabla_{k}\left(M_{j}[\boldsymbol{M},(\boldsymbol{M}, \nabla) \boldsymbol{M}]_{k}\right)+\left(f^{(22)}-f^{(23)}\right) \nabla_{k}\left(M_{k}[\boldsymbol{M},(\boldsymbol{M}, \nabla) \boldsymbol{M}]_{j}\right) .
\end{gathered}
$$

Как и в предыдущем разделе, здесь использована нумерация постоянных $f^{(\alpha)}$ согласно порядку соответствующих им плотностей в формулах (4.2)-(4.7).

Для того чтобы оператор $\mathrm{L}_{j}[\boldsymbol{M}]$ сохранял свойства унимодальности и соленоидальности поля $\boldsymbol{M}(\boldsymbol{x}, t)$, необходимо и достаточно, чтобы для любого дважды непрерывно дифференцируемого псевдовекторного поля $\boldsymbol{M}$ на $\mathbb{R}^{3}$ тождественно выполнялись равенства

$$
\nabla_{j} \mathrm{~L}_{j}[\boldsymbol{M}]=0, \quad M_{j} \mathrm{~L}_{j}[\boldsymbol{M}]=0 .
$$

Проведем анализ возможностей выполнения этих равенств для произвольных полей $\boldsymbol{M}$ из класса $\mathcal{K}_{2}\left(\mathbb{R}^{3}\right) \cap \mathcal{M}_{0}\left(\mathbb{R}^{3}\right)$. Анализ уравнений (6.2) состоит из следующих пунктов I-VII.

I. Первое из равенств (6.2) приводит к следующему уравнению относительно коэффициентов $f^{(\alpha)}$ :

$$
\begin{aligned}
& f^{(7)} \nabla_{j} \nabla_{k}(\left.M_{j}(\boldsymbol{M}, \nabla) M_{k}+M_{k}(\boldsymbol{M}, \nabla) M_{j}\right)+f^{(10)} \Delta(\boldsymbol{M},[\nabla, \boldsymbol{M}])+ \\
&+f^{(11)} \nabla_{j} \nabla_{k}\left([\boldsymbol{M}, \nabla]_{j} M_{k}+[\boldsymbol{M}, \nabla]_{k} M_{j}\right)+f^{(13)} \nabla_{j} \nabla_{k}\left(\varepsilon_{j l n} M_{n} \nabla_{k} M_{l}+\varepsilon_{k l n} M_{n} \nabla_{j} M_{l}\right)+ \\
& \quad+f^{(16)} \nabla_{j} \nabla_{k}\left(M_{j}[\nabla, \boldsymbol{M}]_{k}+M_{k}[\nabla, \boldsymbol{M}]_{j}\right)+f^{(20)} \nabla_{j} \nabla_{k} M_{j} M_{k}(\boldsymbol{M},[\nabla, \boldsymbol{M}])+ \\
& \quad+f^{(22)} \nabla_{j} \nabla_{k}\left(M_{j}[\boldsymbol{M},(\boldsymbol{M}, \nabla) \boldsymbol{M}]_{k}+M_{k}[\boldsymbol{M},(\boldsymbol{M}, \nabla) \boldsymbol{M}]_{j}\right)=0,
\end{aligned}
$$

а второе - к уравнению:

$$
\begin{gathered}
\left(f^{(2)}-f^{(3)}\right) M_{j} \Delta M_{j}+f^{(10)} M_{j} \nabla_{j}(\boldsymbol{M},[\nabla, \boldsymbol{M}])+\varepsilon_{k l n}\left(f^{(13)}-f^{(14)}\right) M_{j} \nabla_{k}\left(M_{n} \nabla_{j} M_{l}\right)+ \\
+\left(f^{(7)}+f^{(8)}\right) M_{j} \nabla_{k}\left(M_{j}(\boldsymbol{M}, \nabla) M_{k}\right)+\left(f^{(7)}-f^{(8)}\right) M_{j} \nabla_{k}\left(M_{k}(\boldsymbol{M}, \nabla) M_{j}\right)+ \\
+\left(f^{(11)}+f^{(12)}\right) M_{j} \nabla_{k}\left([\boldsymbol{M}, \nabla]_{j} M_{k}\right)+\left(f^{(16)}-f^{(17)}\right) M_{j} \nabla_{k}\left(M_{k}[\nabla, \boldsymbol{M}]_{j}\right)+ \\
\quad+\varepsilon_{j k l} f^{(18)} M_{j} \nabla_{k}(\boldsymbol{M}, \nabla) M_{l}+f^{(20)} M_{j} \nabla_{k} M_{j} M_{k}(\boldsymbol{M},[\nabla, \boldsymbol{M}])+ \\
+\left(f^{(22)}+f^{(23)}\right) M_{j} \nabla_{k}\left(M_{j}[\boldsymbol{M},(\boldsymbol{M}, \nabla) \boldsymbol{M}]_{k}\right)+\left(f^{(22)}-f^{(23)}\right) M_{j} \nabla_{k}\left(M_{k}[\boldsymbol{M},(\boldsymbol{M}, \nabla) \boldsymbol{M}]_{j}\right),
\end{gathered}
$$

где в (6.4) учтены тождества

$$
M_{j} \nabla_{k}[\boldsymbol{M}, \nabla]_{k} M_{j}=M_{j} \nabla_{k} M_{j}[\nabla, \boldsymbol{M}]_{k}=\varepsilon_{j l n} M_{j} \nabla_{k}\left(M_{n} \nabla_{k} M_{l}\right)=0 .
$$

Потребуем, чтобы коэффициенты $f^{(\alpha)}$ в этих уравнениях были универсальны, т.е. не зависели от длины вектора $\boldsymbol{M}$. Тогда приведенные два уравнения распадаются на серии уравнений согласно степени поля $\boldsymbol{M}$ во входящих в них слагаемых, а именно, уравнение (6.3) приводит к уравнениям:

$$
\begin{aligned}
f^{(10)} \Delta(\boldsymbol{M},[\nabla, \boldsymbol{M}])+2 f^{(11)} \nabla_{j} \nabla_{k}[\boldsymbol{M}, \nabla]_{j} M_{k}+2 \varepsilon_{j l n} f^{(13)} \nabla_{j} \nabla_{k} M_{n} \nabla_{k} M_{l}+ & \\
& +2 f^{(16)} \nabla_{j} \nabla_{k} M_{j}[\nabla, \boldsymbol{M}]_{k}=0
\end{aligned}
$$

со слагаемыми степени 2 , к уравнению

$$
f^{(7)} \nabla_{j} \nabla_{k} M_{j}(M, \nabla) M_{k}=0
$$

со слагаемым степени 3 ,

$$
f^{(20)} \nabla_{j} \nabla_{k} M_{j} M_{k}(\boldsymbol{M},[\nabla, \boldsymbol{M}])+2 f^{(22)} \nabla_{j} \nabla_{k} M_{j}[\boldsymbol{M},(\boldsymbol{M}, \nabla) \boldsymbol{M}]_{k}=0,
$$

со слагаемым степени 4. Уравнение (6.4) приводит, соответственно, к уравнениям

$$
\left(f^{(2)}-f^{(3)}\right) M_{j} \Delta M_{j}=0 ;
$$




$$
\begin{gathered}
f^{(10)} M_{j} \nabla_{j}(\boldsymbol{M},[\nabla, \boldsymbol{M}])+\varepsilon_{j k l} f^{(18)} M_{j} \nabla_{k}\left((\boldsymbol{M}, \nabla) M_{l}\right)+\varepsilon_{k l n}\left(f^{(13)}-f^{(14)}\right) M_{j} \nabla_{k}\left(M_{n} \nabla_{j} M_{l}\right)+ \\
+\left(f^{(11)}+f^{(12)}\right) M_{j} \nabla_{k}\left([\boldsymbol{M}, \nabla]_{j} M_{k}\right)+\left(f^{(16)}-f^{(17)}\right) M_{j} \nabla_{k}\left(M_{k}[\nabla, \boldsymbol{M}]_{j}\right)=0 \\
\left(f^{(7)}+f^{(8)}\right) M_{j} \nabla_{k}\left(M_{j}(\boldsymbol{M}, \nabla) M_{k}\right)+\left(f^{(7)}-f^{(8)}\right) M_{j} \nabla_{k}\left(M_{k}(\boldsymbol{M}, \nabla) M_{j}\right)=0 \\
f^{(20)} M_{j} \nabla_{k} M_{j} M_{k}(\boldsymbol{M},[\nabla, \boldsymbol{M}])+\left(f^{(22)}+f^{(23)}\right) M_{j} \nabla_{k}\left(M_{j}[\boldsymbol{M},(\boldsymbol{M}, \nabla) \boldsymbol{M}]_{k}\right)+ \\
+\left(f^{(22)}-f^{(23)}\right) M_{j} \nabla_{k}\left(M_{k}[\boldsymbol{M},(\boldsymbol{M}, \nabla) \boldsymbol{M}]_{j}\right)=0 .
\end{gathered}
$$

II. Равенство (6.8) для унимодального поля $\boldsymbol{M}$ возможно только при выполнении условия $\left(\nabla_{k} M_{l}\right)\left(\nabla_{k} M_{l}\right)=0$; это приводит к заключению, что $\boldsymbol{M}=$ const. Тогда $f^{(2)}=f^{(3)}$. В общем случае унимодального соленоидального поля

$$
\nabla_{j} \nabla_{k} M_{j}(\boldsymbol{M}, \nabla) M_{k} \neq 0 .
$$

Тогда из равенства (6.6) следует, что $f^{(7)}=0$. В этом случае, ввиду соотношения

$$
M_{j}\left(\nabla_{k} M_{j}\right) \cdot(\boldsymbol{M}, \nabla) M_{k}=0
$$

из (6.10) следует, что

$$
\left.f^{(8)} M_{j} M_{k} \nabla_{k}(M, \nabla) M_{j}\right)=0 .
$$

Так как в общем положении поля $\boldsymbol{M}$ выражение при коэффициенте $f^{(8)}$ не равно нулю, то $f^{(8)}=0$.

III. Выявим все остальные равные нулю коэффициенты $f^{(\alpha)}$ при условии, что выполняются равенства $(6.5),(6.7),(6.9)$ и (6.11). Найдем связи между оставшимися неустановленными коэффициентами путем линеаризации этих равенств около постоянного поля $\boldsymbol{M}^{(0)}$. Положим в уравнениях $\boldsymbol{M}=\boldsymbol{M}^{(0)}+\boldsymbol{m}$, где, ввиду соленоидальности и унимодальности поля $\boldsymbol{M}$, поле $\boldsymbol{m}$ также является соленоидальным и лежит в плоскости, ортогональной $\boldsymbol{M}^{(0)}$, а в остальном произвольно. Линеаризация указанных уравнений по полю $\boldsymbol{m}$ приводит к следующим равенствам, которые должны тождественно выполняться при его произвольном выборе с учетом ограничений $\left(\boldsymbol{M}^{(0)}, \boldsymbol{m}\right)=0$ и $(\nabla, \boldsymbol{m})=0$ :

$$
\begin{gathered}
\left(f^{(10)}+2 f^{(13)}\right)\left(\boldsymbol{M}^{(0)},[\nabla, \Delta \boldsymbol{m}]\right)=0 \\
\left(f^{(20)}-2 f^{(22)}\right)\left(\boldsymbol{M}^{(0)}, \nabla\right)^{2}\left(\boldsymbol{M}^{(0)},[\nabla, \boldsymbol{m}]\right)=0 \\
\left(f^{(10)}+f^{(13)}-f^{(14)}+f^{(16)}-f^{(17)}+f^{(18)}\right)\left(\boldsymbol{M}^{(0)}, \nabla\right)\left(\boldsymbol{M}^{(0)},[\nabla, \boldsymbol{m}]\right)=0 \\
\left(f^{(20)}-f^{(22)}-f^{(23)}\right) M^{2}\left(\boldsymbol{M}^{(0)}, \nabla\right)\left(\boldsymbol{M}^{(0)},[\nabla, \boldsymbol{m}]\right)=0 .
\end{gathered}
$$

После подстановки $\boldsymbol{m}=\boldsymbol{A} \exp (\boldsymbol{x}, \boldsymbol{k})$ в равенства (6.12)-(6.15), где векторы $\boldsymbol{k}$ и $\boldsymbol{A}$ таковы, что $\left(\boldsymbol{A}, \boldsymbol{M}^{(0)}\right)=0,(\boldsymbol{A}, \boldsymbol{k})=0$, соответственно, получаем в каждом равенстве не равные нулю множители, зависящие от поля $\boldsymbol{m}$. Тогда

$$
\begin{gathered}
f^{(10)}+2 f^{(13)}=0, \quad f^{(20)}=2 f^{(22)}, \quad f^{(22)}=f^{(23)}, \\
f^{(18)}=f^{(13)}+f^{(14)}+f^{(17)}-f^{(16)} .
\end{gathered}
$$

При учете равенств (6.16) уравнение (6.7) принимает вид

$$
f^{(20)}\left(\nabla_{j} \nabla_{k} M_{j} M_{k}(\boldsymbol{M},[\nabla, \boldsymbol{M}])+\nabla_{j} \nabla_{k} M_{j}[\boldsymbol{M},(\boldsymbol{M}, \nabla) \boldsymbol{M}]_{k}\right)=0
$$


IV. После установления связей (6.16), (6.17) произведем дальнейшие упрощения анализируемых уравнений (6.5), (6.7), (6.9), (6.11). Подставим в равенство (6.18) поле

$$
M_{j}=A_{j} \cos (\boldsymbol{x}, \boldsymbol{k})+B_{j} \sin (\boldsymbol{x}, \boldsymbol{k}), \quad \text { где } k_{j} A_{j}=k_{j} B_{j}=A_{j} B_{j}=0,
$$

которое принадлежит многообразию $\mathcal{M}_{0}\left(\mathbb{R}^{3}\right)$. Тогда

$$
(\boldsymbol{M}, \nabla) \boldsymbol{M}=0, \quad(\boldsymbol{M},[\nabla, \boldsymbol{M}])=\varepsilon_{j k l} k_{k} A_{j} B_{l} .
$$

Поэтому (6.18) сводится к равенству

$$
f^{(20)}\left(\nabla_{k} M_{j}\right)\left(\nabla_{j} M_{k}\right)=0=f^{(20)}\left(\operatorname{Sp~A}^{2}\right),
$$

где бесследовая матрица $(\mathrm{A})_{j k} \equiv A_{j k}=\nabla_{j} M_{k}$ может быть выбрана диагональной в фиксированной точке $\boldsymbol{x}=0$ и потому $\mathrm{Sp}^{2} \neq 0$. Следовательно, $f^{(20)}=0$, так что $f^{(22)}=f^{(23)}=0$.

Уравнение (6.5), с учетом (6.16), принимает вид

$$
f^{(13)}\left(\varepsilon_{j l n} \nabla_{j} M_{n} \Delta M_{l}-\Delta(\boldsymbol{M},[\nabla, \boldsymbol{M}])\right)+\nabla_{j} \nabla_{k}\left(f^{(11)}[\boldsymbol{M}, \nabla]_{j} M_{k}+f^{(16)} M_{j}[\nabla, \boldsymbol{M}]_{k}\right)=0 .
$$

Используя условия унимодальности и соленоидальности поля $\boldsymbol{M}$, преобразуем слагаемые, входящие в (6.19):

$$
\begin{gathered}
\nabla_{j} \nabla_{k}[\boldsymbol{M}, \nabla]_{j} M_{k}=\varepsilon_{j l n} B_{l j k} A_{n k}, \quad \nabla_{j} \nabla_{k} M_{j}[\nabla, \boldsymbol{M}]_{k}=\varepsilon_{j l n} A_{n k} B_{l j k}, \\
\varepsilon_{j l n} \nabla_{j}\left(M_{n} \Delta M_{l}\right)-\Delta(\boldsymbol{M},[\nabla, \boldsymbol{M}])=-2 \varepsilon_{j l n}\left[A_{j l} B_{n k k}+A_{k n} B_{l j k}\right],
\end{gathered}
$$

где $B_{l j k} \equiv \nabla_{j} \nabla_{k} M_{l}$ - симметричный по паре последних индексов тензор третьего ранга. Подстановка этих выражений в уравнение (6.19) приводит его к виду

$$
\varepsilon_{j l n}\left[\left(f^{(11)}+f^{(16)}\right) A_{n k} B_{l j k}-2 f^{(13)}\left(A_{k n} B_{l j k}+A_{j l} B_{n k k}\right)\right]=0 .
$$

V. Проанализируем, теперь, возможность выполнимости равенства (6.20) локально в окрестности произвольной фиксированной точке $\boldsymbol{x}_{0}$ при ненулевых значениях коэффициентов. Не ограничивая общности, будем считать, что $\boldsymbol{x}_{0}=0$. Воспользуемся степенным разложением поля $\boldsymbol{M}$ в этой точке:

$$
M_{j}=M_{j}^{(0)}+A_{j k} x_{k}+\frac{1}{2} B_{j k l} x_{k} x_{l}+o\left(|\boldsymbol{x}|^{2}\right) .
$$

Коэффициенты разложения обязаны удовлетворять условиям

$$
\boldsymbol{M}^{2}=M_{j}^{(0)} M_{j}^{(0)}+2 M_{j}^{(0)} A_{j k} x_{k}+\left(A_{j k} A_{j l}+M_{j}^{(0)} B_{j k l}\right) x_{k} x_{l}+o\left(|\boldsymbol{x}|^{2}\right)=M^{2}
$$

и, следовательно,

$$
2 M_{j}^{(0)} A_{j k} x_{k}+A_{j k} A_{j l} x_{k} x_{l}+M_{j}^{(0)} B_{j k l} x_{k} x_{l}+o\left(|\boldsymbol{x}|^{2}\right)=0 .
$$

Кроме того, должно выполняться соотношение

$$
\nabla_{j} M_{j}=A_{j j}+B_{j j k} x_{k}+o(|\boldsymbol{x}|)=0 .
$$

Тогда для возможности существования разложения (6.21) необходимо и достаточно, чтобы коэффициенты $A_{j k}$ и $B_{j k l}$ удовлетворяли следуюшим условиям:

$$
B_{j k l}=B_{j l k}, \quad A_{j j}=0, \quad M_{j}^{(0)} A_{j k}=0, \quad B_{j j k}=0, \quad A_{j k} A_{j l}+M_{j}^{(0)} B_{j k l}=0,
$$

а в остальном они могут быть выбраны произвольно. 
VI. Докажем, что $f^{(13)}=0$. Не ограничивая общности, считаем, что $M_{3}^{(0)}=M$. Тогда из (6.22) следует, что

$$
A_{j 3}=0, \quad A_{m k} A_{m l}+M B_{3 k l}=0, \quad k, l=1,2,3 .
$$

Положим $A_{k l}=\delta_{k 3}\left(1-\delta_{l 3}\right)$. Тогда

$$
\left(\mathrm{A}^{T} \mathrm{~A}\right)_{k l}=\left(1-\delta_{k 3}\right)\left(1-\delta_{l 3}\right)
$$

и, ввиду (6.22), $B_{3 k l}=0$, если хотя бы один из индексов равен 3 , и $B_{3 k l}=-M^{-1}$ при $k, l \neq 3$.

Элементы $B_{j k l}$, где $j=1,2$, могут быть выбраны произвольно, но с учетом соотношений

$$
B_{j j k}=0, \quad B_{j k l}=B_{j l k} .
$$

Положим

$$
B_{j k l}=b^{(j)} B_{k l}, \quad j=1, \quad k, l=1,2,3,
$$

где матрица $B_{k l}$ симметрична. Рассмотрим условие $B_{j j k}=0$. Так как $B_{33 k}=0$, то при $k=3$ получаем

$$
B_{113}+B_{223}=b^{(1)} B_{13}+b^{(2)} B_{23} \equiv b^{(1)} b_{1}+b^{(2)} b_{2}=0 .
$$

Удовлетворить этому равенству для любых наперед заданных чисел $b^{(1)}$ и $b^{(2)}$ всегда можно, подбирая подходящим образом числа $b_{1}$ и $b_{2}$.

При $k=1,2$ условие $B_{j j k}=0$ превращается в

$$
b^{(1)} B_{1 k}+b^{(2)} B_{2 k}=0,
$$

т.е. $B_{2 k}=\lambda B_{1 k}$, где $\lambda=-b^{(1)} / b^{(2)} \in \mathbb{R}-$ произвольное число. Наконец, из условия симметрии матрицы $B_{k l}$ следует, что

$$
B_{k l}=b\left(\begin{array}{cc}
1 & \lambda \\
\lambda & \lambda^{2}
\end{array}\right), \quad b=B_{11}
$$

Используя определенные выше тензоры $A_{j k}$ и $B_{j k l}$, находим, что при $b^{(1)}=b^{(2)}$ имеют место равенства

$$
\varepsilon_{j l n} A_{n k} B_{l j k}=b b^{(2)}\left(1-\lambda^{2}\right), \quad \varepsilon_{j l n} A_{j l} B_{n k k}=0, \quad \varepsilon_{j l n} A_{k n} B_{l j k}=0,
$$

где для значений еще не определенных коэффициентов $B_{k 33}, k=1,2$, мы положили $B_{133}=B_{233}$. Подставляя вычисленные значения в $(6.20)$, находим $2 b b^{(2)}\left(1-\lambda^{2}\right) f^{(13)}=0$, откуда следует $f^{(13)}=$ 0 .

VII. Так как $f^{(13)}=0$, то из (6.20) следует $f^{(11)}=-f^{(16)}$ ввиду того, что $\varepsilon_{j l n} A_{n k} B_{l j k}$ не равно нулю в общем случае. Учитывая это равенство вместе с $f^{(13)}=0$, а также равенства (6.16) и (6.17), запишем уравнение (6.9) в следующей форме:

$$
\begin{gathered}
f^{(12)} M_{j} \nabla_{k}[\boldsymbol{M}, \nabla]_{j} M_{k}-f^{(14)} M_{j} \nabla_{k}\left(\varepsilon_{k l n} M_{n} \nabla_{j} M_{l}-\varepsilon_{j k l}(\boldsymbol{M}, \nabla) M_{l}\right)+ \\
+f^{(16)} M_{j} \nabla_{k}\left(M_{k}[\nabla, \boldsymbol{M}]_{j}-[\boldsymbol{M}, \nabla]_{j} M_{k}-\varepsilon_{j k l}(\boldsymbol{M}, \nabla) M_{l}\right)- \\
\quad-f^{(17)} M_{j} \nabla_{k}\left(M_{k}[\nabla, \boldsymbol{M}]_{j}-\varepsilon_{j k l}(\boldsymbol{M}, \nabla) M_{l}\right)=0 .
\end{gathered}
$$

Воспользовавшись соленоидальностью и унимодальностью поля $\boldsymbol{M}$ и вводя обозначения $A_{j k}=\nabla_{j} M_{k}, B_{j k l}=\nabla_{k} \nabla_{l} M_{j}$, посредством простых преобразований в рамках тензорной алгебры приведем это уравнение к виду

$$
\left(f^{(12)}-f^{(14)}-f^{(17)}\right) \varepsilon_{k l m} M_{l} A_{k j} A_{j m}-f^{(14)} \varepsilon_{j k m} M_{l} A_{k j} A_{l m}-2 f^{(16)} \varepsilon_{j l m} M_{j} M_{k} B_{m k l}=0,
$$

в котором в левой части представлено разложение по линейно независимым инвариантам, составленным из тензоров $A_{j k}, B_{j k l}$ и псевдовектора $M_{j}$. Из полученного уравнения, которое должно выполняться для любого соленоидального унимодального дважды непрерывно дифференцируемого поля $\boldsymbol{M}$ на $\mathbb{R}^{3}$, с необходимостью следует, что

$$
f^{(16)}=0, \quad f^{(14)}=0, \quad f^{(12)}=f^{(17)} .
$$


Введя обозначение $f^{(12)}=\gamma$ и учтя все полученные в процессе проведения анализа ограничения на выбор коэффициентов $f^{(\alpha)}$, приходим на основании (6.1) к следующему общему виду дифференциальных операторов класса $\mathcal{K}_{2}\left(\mathbb{R}^{3}\right) \cap \mathcal{M}_{0}\left(\mathbb{R}^{3}\right)$ :

$$
\mathrm{L}_{j}[\boldsymbol{M}]=\gamma \nabla_{k}\left([\boldsymbol{M}, \nabla]_{j} M_{k}-[\boldsymbol{M}, \nabla]_{k} M_{j}+M_{j}[\nabla, \boldsymbol{M}]_{k}-M_{k}[\nabla, \boldsymbol{M}]_{j}+\varepsilon_{j k l}(\boldsymbol{M}, \nabla) M_{l}\right) .
$$

Таким образом, доказана следующая основная теорема, дающая описание класса $\mathcal{K}_{2}\left(\mathbb{R}^{3}\right) \cap \mathcal{M}_{0}\left(\mathbb{R}^{3}\right)$ для псевдовекторного поля.

Теорема 6.1. Линейное многообразие $\mathcal{K}_{2}\left(\mathbb{R}^{3}\right) \cap \mathcal{M}_{0}\left(\mathbb{R}^{3}\right)$ всех дифференциалъных уравнений

$$
\dot{M}_{j}=\mathrm{L}_{j}[\boldsymbol{M}]
$$

дивергентного типа для псевдовекторных соленоидальных унимодалъных полей $\boldsymbol{M}$ на $\mathbb{R}^{3}$ из пространства $\left[C_{2, \text { lос }}\left(\mathbb{R}^{3}\right)\right]^{3}$ с дифференциальными операторами $\mathrm{L}_{j}[\boldsymbol{M}]$ второго порядка, действующими в этом пространстве, ковариантных относительно действия преобразований группы $\boldsymbol{O}_{3}$, которые оставляют инвариантным любое многообразие

$$
\left\{\boldsymbol{M}:(\nabla, \boldsymbol{M})=0 ; \boldsymbol{M}^{2}=M^{2}, \boldsymbol{x} \in \mathbb{R}^{3}\right\} \subset\left[C_{2, \mathrm{loc}}\left(\mathbb{R}^{3}\right)\right]^{3}
$$

в этом пространстве с произвольным значением $M^{2} \in(0, \infty)$, одномерно. Это линейное многообразие описывается формулой (6.23) с произвольной постоянной $\gamma \in \mathbb{R}$.

7. Заключение. Идея, на которой основано проведенное исследование, связана с проблемой описания на макроскопическом уровне динамики сложным образом устроенных конденсированных сред. Полученный в работе конкретный результат связан с известной в физике магнетизма проблемой построения адекватного эволюционного уравнения для поля $\boldsymbol{M}$ плотности намагниченности, обладающего необратимой динамикой (см. [1-3,5]). Известно эволюционное уравнение Ландау-Лифшица (см., например, $[9,10])$, которое в изученном в работе сферически симметричном случае имеет вид

$$
\dot{M}=\gamma[\boldsymbol{M}, \Delta \boldsymbol{M}]
$$

Оно имеет дивергентный тип, так как

$$
[\boldsymbol{M}, \Delta \boldsymbol{M}]_{j}=\nabla_{k} \varepsilon_{j l n} M_{l} \nabla_{k} M_{n},
$$

очевидным образом сохраняет унимодальность поля, но не сохраняет соленоидальность и, что более существенно, не обладает «диссипацией». Это связано с тем, что собственные числа $(3 \times$ $3)$-матрицы, представляющей символ линеаризации оператора $L_{j}[\boldsymbol{M}]$, не имеют вещественной части. Попытки устранения указанного недостатка эволюционного уравнения ферродинамики, основанные на обобщении уравнения Ландау-Лифшица, не является, на наш взгляд, удачными (см., например, $[2,3]$ ). Так как оператор $\mathrm{L}_{j}[\boldsymbol{M}]=\gamma[\boldsymbol{M}, \Delta \boldsymbol{M}]$ не содержится среди слагаемых в правой части эволюционного уравнения

$$
\dot{M}_{j}=\gamma \nabla_{k}\left([\boldsymbol{M}, \nabla]_{j} M_{k}-[\boldsymbol{M}, \nabla]_{k} M_{j}+M_{j}[\nabla, \boldsymbol{M}]_{k}-M_{k}[\nabla, \boldsymbol{M}]_{j}+\varepsilon_{j k l}(\boldsymbol{M}, \nabla) M_{l}\right)
$$

с оператором $L_{j}[\cdot]$, определяемым формулой (6.23), то теорема 6.1 показывает, с чем связано такое положение.

Заметим, что символ линеаризации оператора, представленного формулой (6.23), ввиду наличия двух инвариантов движения, необходимо имеет два нулевых собственных числа. Поэтому его третье собственное число обязательно вещественно. Выбором же подходящего знака постоянной $\gamma$ можно добиться того, что этот оператор будет иметь параболический тип.

В работе рассмотрены только лишь простейшие постановки задач из числа тех, связанные с неприводимыми векторными представлениями и которые могли бы представлять интерес для физических приложений. Очевидное обобщение изученных задач состоит в отказе от сферической симметрии, т.е. в использовании при построении всех возможных тензорных коэффициентов как в векторном, так и в пседовекторном случае тензорной алгебры с расширением множества образующих за счет тензора второго ранга, характеризующего асимметрию среды. При такой постановке задачи возникает существенно более богатое множество тензорных коэффициентов, 
определяющих плотности $S_{j ; k}, j=1,2,3$, что приводит к более трудоемкому анализу всех представляющихся возможностей.

Другой путь обобщений состоит в отказе от выполнения сразу двух условий: унимодальности и соленоидальности. В этом случае классы допустимых уравнений становятся намного более широкими. В частности, в первом случае для псевдовекторного поля такой класс содержит уже упомянутое уравнение Ландау-Лифшица, а во втором - известное уравнение Навье-Стокса для несжимаемой жидкости.

Наконец, самые широкие обобщения постановки задачи, которая решена в работе, усматриваются из ее общей постановки, изложенной в разделе 2. Более того, с точки зрения приложений имеет смысл изучение эволюционных уравнений с дифференциальными операторами, которые не являются дивергентными, но которые представимы в виде

$$
\dot{X}_{a}=\left(\nabla_{k} S_{a ; k}\right)(X)+H_{a}(X), \quad a=1, \ldots, N,
$$

где $H_{a}(X)$ - функция на пространстве значений полного набора локальных термодинамических параметров, ковариантно преобразующаяся при действии преобразований группы $\mathbf{O}_{3}$ и служащая с физической точки зрения «самосогласованным источником» поля.

\section{СПИСОК ЛИТЕРАТУРЫ}

1. Андреев А. Ф., Марченко В. И. Макроскопическая теория спиновых волн// ЖЭТФ. - 1976. - 70, № $4 .-$ C. $1522-1532$.

2. Барълхтар В. Г. Жизнь в науке. - Киев: Наукова думка, 2010.

3. Барълхтар В. Г., Белых В. Г., Соболева Т. К. Макроскопическая теория релаксации коллективных возбуждений в неупорядоченных и неколлинеарных магнетиках// Теор. мат. физ. -1988 . -77 , № 2. - C. 311-318.

4. Вирченко Ю. П., Понамарева А. Э. Построение общего эволюционного уравнения для псевдовекторного соленоидального поля с локальным законом сохранения// Науч. вед. Белгород. гос. ун-та. Сер. Мат. Физ. - 2018. - 50, № 2. - С. 224-232.

5. Волков Д. В. Феноменологические лагранжианы// Физ. элем. част. атом. яд. $-1973 .-4$, № 1. C. $3-41$.

6. Гилбарг Д., Трудингер Н. Эллиптические дифференциальные уравнения с частными производными второго порядка. - М.: Наука, 1989.

7. Исаев А. А., Ковалевский М. Ю., Пелетминский С. В. Гамильтонов подход в теории конденсированных сред со спонтанно нарушенной симметрией// Физ. элем. част. атом. яд. $-1996 .-27$, № 2 . C. 431-492.

8. Кач, Е. И., Лебедев В. В. Динамика жидких кристаллов. - М.: Наука, 1988.

9. Косевич А. М., Иванов Б. А., Ковалев А. С. Нелинейные волны намагниченности. - Киев: Наукова думка, 1988.

10. Ландау Л. Д., Лифшиц, Е. М. К теории дисперсии магнитной проницаемости ферромагнитных тел// в кн.: Ландау Л. Д. Собрание трудов. - М.: Наука, 1969. - Т. 1. - С. 128.

11. Любарский Г. Я. Теория групп и ее приложения в физике. - М.: ГИФМЛ, 1958.

12. Мак-Коннел А. Дж. Введение в тензорный анализ с приложениями к геометрии, механике и физике. - М.: ГИФМЛ, 1963.

13. Рашевский П. К. Риманова геометрия и тензорный анализ. - М.: Наука, 1967.

14. Субботин A. В. Описание класса эволюционных уравнений дивергентного типа для векторного поля// Науч. вед. Белгород. гос. ун-та. Сер. Мат. Физ. - 2018. - 50, № 4. - С. 492-497.

15. Briceyda B. D., Porter R. M. General Solution of the Inhomogeneous Div-Curl System and Consequences. - Adv. Appl. Clifford Alg., 2017.

Вирченко Юрий Петрович

Белгородский государственный университет

E-mail: virch@bsu.edu.ru

Субботин Андрей Валерьевич

Белгородский государственный университет

E-mail: Subbotin@bsu.edu.ru 